not improved by operation further emboli occurred in two, and two had emboli for the first time during the follow-up years. Three patients from grades 2 and 3 who had preoperative emboli and two who had not had pre-operative emboli had emboli at operation. None of the patients who had good operative results had post-operative emboli. Thus, counting both the emboli at operation and during the three follow-up years, 10 of the 84 patients reviewed had emboli (12\%).

Bannister (1960) during an average of 3.4 years' follow-up reported an incidence of systemic emboli of $36 \%$ in patients with mitral stenosis over 40 , and an overall mortality of $5 \%$ ( $1.5 \%$ per year). If our patients did not have mitral valvotomy, 30 patients would have been expected to have emboli over a period of three years (36\%). Our data suggests a reduction in the incidence of post-operative emboli, since 10 patients $(12 \%)$ had further emboli. This is, however, associated with an operative mortality of $6 \%$, a further mortality of $5 \%$ per year, and poor operative results, particularly in the group of patients who were in pre-operative grades 0 and 1 and might have been expected to do well.

Significant mitral stenosis remains the indication for operation. Our results show that the occurrence of systemic emboli is not an additional reason for mitral valvotomy. In patients over 40 who have emboli, but only minimal mitral stenosis, anticoagulant treatment might be considered, although this carries its own difficulties and risks. Szekely (1964) reports a reduced incidence of emboli from $9.4 \%$ to $3.4 \%$ per year with anticoagulant treatment. One might also consider removal of the atrial appendage without interfering with the mitral valve as an alternative procedure.

\section{Summary}

A three-year follow-up is reviewed of 84 patients over the age of 40 who had mitral valvotomy at Guy's Hospital.

The immediate mortality of mitral valvotomy was $6 \%$, with a further mortality of $5 \%$ per year during the follow-up.

The results in 34 patients who had had previous systemic emboli showed only $27 \%$ good results in respect of exercise tolerance compared with $47 \%$ in the non-emboli group.

Thirteen patients who had minimal mitral stenosis and were operated on to prevent further emboli also had poor results.

Six of the 84 patients had further emboli at operation and a further four had emboli during the three-year follow-up.

The occurrence of emboli is not thought to be an additional reason for performing mitral valvotomy, and long-term anticoagulant treatment might be used.

We are indebted to Sir Russell Brock, Mr. Donald Ross, and Mr. R. H. F. Brain, who operated on these patients, and to Dr. Charles Baker and Dr. Ralph Kauntze, who allowed us to use their records. We are particularly grateful to Dr. Charles Baker for his advice, help, and contribution to this paper.

\section{REFERENCES}

Baker, C., Brock, R. C., Campbell, M., and Wood, P. (1952). Brit. med. 7., 1, 1043. and Hancock, W. E. (1960). Brit. Heart 7., 22, 281.

Bannister, R. G. (1960). Lancet, 2, 329.

Gilchrist, A. R. (1963). Brit. med. 7., 1, 209.

Szekely, P. (1964). Ibid., 1, 1209 .

Szekely, P. (1964). Ibid., 1, 1209.

\title{
Studies on Tyrosinosis : 1, Effect of Low-tyrosine and Low-phenylalanine Diet
}

\author{
SVERRE HALVORSEN,* M.D. ; LEIV R. GJESSING, † M.D.
}

Brit. med. F., 1964, 2, 1171-1173

It has repeatedly been stated that no proved case of tyrosinosis has been described since the original report of Medes (1932) on a 49-year-old man with myasthenia gravis who excreted large amounts of $p$-hydroxyphenyl-pyruvic acid, the $\alpha$-keto-acid of tyrosine, in the urine. Sakai and Kitagawa (1957a, 1957b) and Sakai et al. (1959) reported, however, on a child with hepatomegaly, splenomegaly, liver dysfunction, and progressive hypophosphataemic rickets who subsequently died of liver cirrhosis with hepatoma. This child excreted large amounts of p-hydroxyphenyl-lactic acid and moderate amounts of $p$-hydroxyphenyl-pyruvic acid and $p$-hydroxyphenyl-acetic acid together with tyrosine in the urine. The authors postulated a lack of the p-hydroxyphenyl-pyruvate oxidase and reported their patient as an atypical case of tyrosinosis. Since that time similar cases have been reported by Zetterström (1963) and Fritzell et al. (1964).

In June 1963 a 1-year-old girl was admitted to the Children's Department, Rikshospitalet, Oslo, because of hepatomegaly. She was found to have hypophosphataemic rickets, elevated serum-tyrosine level, amino-aciduria with pronounced tyrosine

* Paediatric Research Institute, Rikshospitalet, Oslo.

t Central Laboratory, Dikemark Hospital, Asker, Norway. Financial support from U.S. Public Health Service Grant MH-05726-03, is gratefully acknowledged. excretion, and a large urinary excretion of $p$-hydroxyphenylpyruvic acid, $p$-hydroxyphenyl-lactic acid, and $p$-hydroxyphenyl-acetic acid. The excretion of the tyrosine metabolites was unchanged after loading with vitamin C. Three similar cases have been diagnosed since that time and a detailed report of all these cases will be published elsewhere.

The ultimate proof that this disease is due to a deficiency of p-hydroxyphenyl-pyruvate oxidase is still lacking. Further, the pathogenesis of the liver and kidney dysfunction and of the other symptoms and signs of this disease remains obscure. Nor has any specific therapy been available. It was thought that a trial with a low-tyrosine and low-phenylalanine diet might give an answer to some of these questions, and it is the purpose of the present paper to report briefly the clinical and biochemical findings in our first case and to describe the effects of low-tyrosine and low-phenylalanine diet on the excretion of tyrosine and its metabolites, on the liver and kidney function, and on the general clinical condition of the patient.

\section{Methods}

The serum tyrosine and phenylalanine were determined according to the method of Berry (1962) except that butanol/ 
acetic-acid/water $(60: 15: 25)$ was used as solvent and with equally good separation for tyrosine as for phenylalanine. The urinary amino-acids were determined by two-dimensional paper chromatography. The $p$-hydroxyphenyl-lactic acid and $p$-hydroxyphenyl-acetic acid were determined semiquantitatively according to Armstrong et al. (1956). The $p$-hydroxyphenylpyruvic acid was tested only with ferric chloride and 2-4dinitrophenylhydrazine (D.P.H.) tests. The tubular reabsorption of phosphorus (T.R.P.) was calculated from the serum and urine creatinine and phosphorus as described by Goldman et al. (1957). The prothrombin and proconvertin were determined by the P.P. method of Owren and Aas (1951).

\section{Case Report}

The patient, a girl born on 26 May 1962, was the first child of healthy unrelated parents. The pregnancy, delivery, and neonatal period were normal. She had received adequate food with supplements of vitamins $C$ and $D$.

At the age of 6 months it was noticed that her abdomen was large. She had frequent vomits, and on her admission to a local hospital for pneumonia, rickets, hepatomegaly, and enlarged kidneys were found. When admitted to the Children's Department, Rikshospitalet, Oslo, in June 1963, she was pale, with blue eyes and blond hair. The musculature was hypotonic and the liver, spleen, and kidneys were enlarged. The main laboratory findings are shown in the Table and the Chart. Further, the N.P.N. and creatinine were normal, thymol 0.15 unit, S.G.O.T. 79 and 80 units; there was no Bromsulphalein retention. The serum potassium was 3.6 $\mathrm{mEq} / 1$., sodium $148 \mathrm{mEq} / \mathrm{l}$., and chlorides $119 \mathrm{mEq} / 1$. Her stools were voluminous with increased fat excretion. The sweat electrolytes were normal.

The urine showed slight proteinuria and a positive Benedict's reaction. Galactose, fructose, and lactose were identified on chromatography. There was a moderate increase of leucocytes and a constant increase in renal epithelial cells. The ferric chloride test was positive (green) and the D.P.H. test positive. Amino-acid chromatography revealed a moderate generalized hyperaminoaciduria with a pronounced tyrosine spot. The serum tyrosine level was elevated without increase in other amino-acids, indicating a moderate renal hyperaminoaciduria with an at least partial overflow of tyrosine. Studies of tyrosine metabolites showed an enormous excretion of $p$-hydroxyphenyl-lactic acid $(12,000 \mu \mathrm{g} . / \mathrm{mg}$. creatinine $)$ and a large excretion of $p$-hydroxyphenyl-acetic acid $(4,000 \mu \mathrm{g} . / \mathrm{mg}$. creatinine). These findings indicated a block or inhibition of the oxidation of $p$-hydroxyphenyl-pyruvic acid to homogentisic acid, and it was decided to try a phenylalanine- and tyrosine-restricted diet.

The patient was controlled in December 1963 and had almost the same findings as previously, the serum phosphorus, however, being definitely lower $(1.2 \mathrm{mg} . / 100 \mathrm{ml}$.). In April 1964 she was readmitted for a trial of a low-tyrosine diet. She showed definite clinical and radiological signs of rickets. The liver, spleen, and kidneys were unchanged on palpation, the musculature was hypotonic, but she was otherwise in relatively good condition. She had gross excretion of renal epithelial cells and renal casts. The main laboratory findings are shown in the Table and the Chart.
Before the start of the low-tyrosine and low-phenylalanine diet she was in hospital for one week and received the regular hospital diet with about $3,200 \mathrm{mg}$. of phenylalanine plus tyrosine daily. The low-tyrosine diet was given as Cymogran without added tyrosine. She received $150 \mathrm{~g}$. of Cymogran without tyrosine daily in three divided portions, and took the diet remarkably well. Small quantities of milk were given to supply her with phenylalanine and tyrosine, starting with about $45 \mathrm{mg}$. of each and reducing them to about $25 \mathrm{mg}$. per $\mathrm{kg}$. body weight per day. In addition selected fruits, vegetables, starch, and cream were given as in the diet for phenylketonurics. She had received 3,000 I.U. of vitamin D daily during the last five months and remained on this dose throughout the study. Further, during the same time she had been receiving a vitamin $B$ and $C$ preparation providing $75 \mathrm{mg}$. of ascorbic acid daily, and this dose was doubled two weeks prior to the diet and kept throughout the dietary period.

The urine was checked daily with ferric chloride, D.P.H., and microscopical examinations. The ferric chloride test soon became negative, the D.P.H. became negative somewhat later, and the number of renal epithelial cells and casts decreased. One morning the ferric chloride test was again positive, and it was found that she had received at least $200 \mathrm{ml}$. of milk by mistake the day before. The serum tyrosine level dropped from $6-7$ to $1.5 \mathrm{mg} . / 100 \mathrm{ml}$., the serum phosphorus increased from 1.3 to $3 \mathrm{mg} . / 100 \mathrm{ml}$., and the T.R.P. rose from 7 to $96 \%$ (see Chart). The amino-acid chromatograms changed to normal except that methionine and tryptophan were more prominent than normally, probably because of the DL-methionine and DL-tryptophan addition to Cymogran, as is also seen in phenylketonurics on diet. The excretion of $p$-hydroxyphenyl-lactic acid and $p$-hydroxyphenyl-acetic acid was gradually brought to normal (see Table).

The standard bicarbonate, the $\mathrm{pH}$, and the thrombocytes rose slightly but remained below normal levels. The P.P. remained unchanged throughout the study.

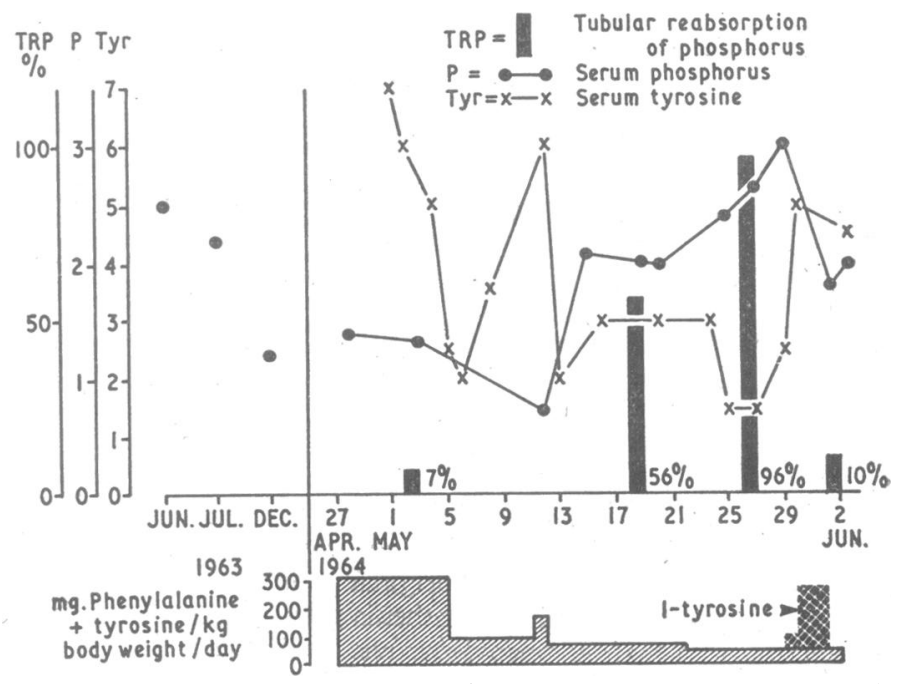

Effects of tyrosine-phenylalanine restricted diet on serum tyrosine (mg./ $100 \mathrm{ml}$. ), serum phosphorus (mg. $/ 100 \mathrm{ml}$.), and tubular reabsorption of phosphorus in a case of tyrosinosis.

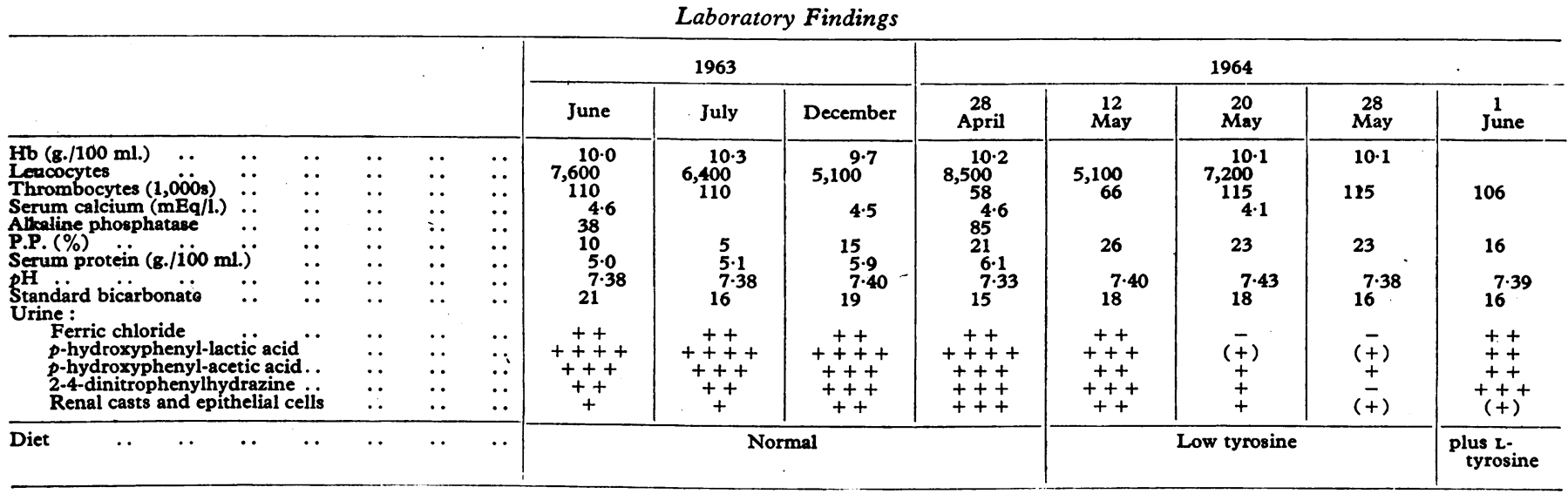


When L-tyrosine alone was added to the diet $(0.6 \mathrm{~g}$. the first day and $2.25 \mathrm{~g}$. the next two days) the fasting serum tyrosine increased to $5 \mathrm{mg} . / 100 \mathrm{ml}$., the ferric chloride and D.P.H. tests in the urine got strongly positive, the serum phosphorus dropped to 1.8 and $2 \mathrm{mg} . / 100 \mathrm{ml}$., and the T.R.P. decreased to $10 \%$ (see Chart). The excretion of the amino-acids and the p-hydroxyphenolic acids increased again to levels as before the diet (see Table).

The mother, who stayed with the child during the dietary period, thought her child was more active and alert during the last part of the low-tyrosine regimen than she had ever been, but this was not apparent to other observers.

\section{Discussion}

The clinical disorder described in this paper and previously reported by Sakai and Kitagawa (1957a, 1957b), Sakai et al. (1959), Zetterström (1963), and Fritzell et al. (1964) has characteristic clinical, biochemical, and morphological findings, and it is our opinion that it represents a disease entity. Although the ultimate proof is still lacking, the previously reported findings and the data here presented support the hypothesis that the disorder is caused by a deficiency of p-hydroxyphenyl-pyruvate oxidase. An inhibition of this enzyme rather than a genetically determined lack cannot be excluded, but we accept Zetterström's view that the disease may be termed "tyrosinosis" rather than atypical tyrosinosis as called by Sakai et al.

The results of the low-tyrosine diet in the above case indicate that the high levels of tyrosine and/or its metabolites are of fundamental importance for the development of the disease. The relation between the levels of tyrosine and its metabolites and the renal handling of phosphorus and amino-acids suggests a causal effect. Although it may be argued that the increased phosphorus reabsorption on a low-tyrosine diet could be caused by extrarenal factors, the simultaneous increase in amino-acid reabsorption indicates that it has had an effect on the renal tubules. The definite decrease in renal epithelial cells and renal casts also supports this assumption. The mechanism by which tyrosine or its metabolites may increase phosphorus and aminoacid excretion remains unknown, but it is likely that the tubular transport of these compounds is inhibited. It is also interesting that glucose transport is less affected, since the patient only occasionally had glycosuria.

The effect of the low-tyrosine diet on the thrombocytes is less convincing, and in this short trial the liver function has not been affected, judged by the P.P. test. This was, however, not expected, since the patient already had a far-advanced liver cirrhosis. Although the present study was promising, the ultimate effect of the low-tyrosine diet in this disease cannot yet be assessed. We must await further trials, both long-term and earlier in life, before liver damage has occurred. These preliminary results are published in order to stimulate others to try the diet in such cases.

The above study indicates that the intake of phenylalanine and tyrosine must be low in order to normalize the metabolism. An intake of phenylalanine and tyrosine of about $25 \mathrm{mg}$. of each per kg. body weight per day lowered serum tyrosine to $1.5 \mathrm{mg} . / 100 \mathrm{ml}$., and it was at this level that the improvement in renal function first became convincing.

\section{Summary}

A case of tyrosinosis in a 2-year-old girl is described. The serum-tyrosine level was raised and the urinary excretion of p-hydroxyphenyl-pyruvic acid, p-hydroxyphenyl-lactic acid, and $p$-hydroxyphenyl-acetic acid was much increased. She had marked hyperphosphaturia and hyperaminoaciduria. A four-weeks trial with a low-tyrosine and low-phenylalanine diet decreased the serum-tyrosine level while the serum-phosphorus values increased. The excretion of the tyrosine metabolites, amino-acids, and phosphorus in the urine decreased and the excretion of renal epithelial cells and renal casts diminished. On adding L-tyrosine to the low-tyrosine and low-phenylalanine diet the serum tyrosine again increased while the serum phosphorus decreased, and there was an increase in the excretion of the tyrosine metabolites, amino-acids, and phosphorus in the urine. These findings support the hypothesis that this disease is caused by a defect of $p$-hydroxyphenyl-pyruvate oxidase, and indicate that tyrosine or its metabolites have a " toxic" effect on renal tubular cells and possibly on liver cells.

\section{REFERENCES}

Armstrong, M. D., Shaw, K. N. F., and Wall, P. E. (1956). F. biol. Chem., 218, 293.

Berry, H. K. (1962). Clin. Chem., 8, 172.

Fritzell, S., Jagenburg, O. R., and Schnürer, L. B. (1964). Acta paediat. (Stockh.), 53, 18.

Goldman, L., Gordan, G. S., and Chambers, E. L. (1957). Ann. Surg., $146,407$.

Medes, G. (1932). Biochem. 7., 26, 917.

Owren, P. A., and Aas, K. (1951). Scand. f. clin. Lab. Invest., 3, 201.

Sakai, K., and Kitagawa, T. (1957a). fikeikai med. f., 4, 1.

- (1957b). Ibid., 4, 11.

Zetterström, R. (1963). Ann. N.Y. Acad. Sci., 11i, 220.

\title{
Evaluation of Immunofluorescent and Direct Agglutination Methods for Detection of Specific Toxoplasma Antibodies
}

\author{
J. D. FULTON,*† M.A., M.B., D.T.M. ; A. VOLLER, ${ }^{*} \ddagger$ M.SC., PH.D.
}

A number of serological tests have been described for the detection of antibodies to Toxoplasma gondii, the most recent being the fluorescent antibody test (F.A.T.). (Goldman, 1957 ; Kelen et al., 1962 ; Mandras et al., 1962 ; Camargo, 1964 ; Kramarzh, 1963). We had at our disposal a number of sera whose titres had been determined by the dye test (Sabin and Feldman, 1948), the complement-fixation test (C.F.T.) (Fulton and Dumbell, 1949), and the direct agglutination test (D.A.T.) (Fulton and Turk, 1959). It was possible, therefore, to compare the results with those determined by the newer method.

\section{Materials and Methods}

The antigen, prepared by the method of Fulton and Turk (1959), was a suspension of $T$. gondii free from other cells, except an occasional red cell. Smears were made on microscope slides, which were then dried for several hours in a desiccator before being transferred for storage at $-70^{\circ} \mathrm{C}$. These smears

* London School of Hygiene and Tropical Medicine.

† Supported by the Medical Research Council.

f Supported by the World Health Organization. 